Reprod. Nutr. Dévelop., 1980, 20 (5 B), 1625-1632.

\title{
Analyse de la séquence alimentaire du porc, du sevrage à l'état adulte
}

\author{
par P. AUFFRAY, J. C. MARCILLOUX \\ avec la collaboration technique de L. ROYER, de A. BROUILLE * et de J. L. PIERRE ** \\ Laboratoire de Physiologie de la Nutrition. \\ * Station de Physiologie Animale \\ I.N.R.A., 78350 Jouy en Josas, France. \\ ** Laboratoire de Physiologie des Centres Nerveux, Université Paris VI
}

Summary. Analysis of porcine feeding patferns from weaning to adulthood.

This study describes the instruments used in recording the feeding patterns of 21 pigs of three different breeds fed ad libitum from weaning to adulthood. The feeding behavior of the pigs was characterized by 1) the presence of well individualized meals, and 2) the circadian distribution of food intake : whatever the breed, the animals preferred to eat in the daytime. This diurnal character of food intake was accentuated gradually with age. The frequency of the daily food intake decreased progressively from a mean of 9.5 meals after weaning to usually 3 at adulthood when growth was finished ( 2 meals in the daytime and 1 at night).

\section{Introduction.}

Nous disposons de très peu de données sur la prise alimentaire du porc. Elles résultent le plus souvent de travaux entrepris dans un but appliqué immédiat (Fowler, Mc Donald et Robb, 1971 ; Montgomery et al., 1978). Les mécanismes de régulation physiologique du comportement alimentaire ont été très peu étudiés chez cette espèce. Les recherches effectuées dans ce domaine sur les animaux de laboratoire (rat), c'est-d̀-dire le rôle des stimuli humoraux dans l'initiation ou l'arrêt d'un repas, ef le rôle des différentes structures nerveuses impliquées dans les processus de faim ou de satiété, viennent seulement d'être abordés chez le porc (Auffray, 1969 ; Khalaf, 1969).

Le présent travail a pour objectif d'analyser la séquence alimentaire du porc du sevrage à l'état adulte, et de rechercher dans quelle mesure elle obéit aux règles décrites chez le rat.

\section{Matériel et méthodes.}

1. Dispositifs d'enregistrement.

Plusieurs types d'appareils ont été mis au point, la description de certains ayant été faite par ailleurs, nous nous bornerons à énumérer ici leurs principales carac- 
téristiques (Auffray, Bahy et Marcilloux, 1974). Ces dispositifs ont été conçus dans le but d'étudier « le profil alimentaire » (feeding pattern) de porcs de trois races différentes : Large White, Piétrain, depuis le sevrage jusqu'à l'état adulte (poids vif $8-10 \mathrm{~kg}$ à $200-250 \mathrm{~kg}$ ) et des porcs miniatures de Göttingen adultes (poids vif $70 \mathrm{~kg}$ à $90 \mathrm{~kg}$ ).

A) Dispositif d'enregistrement du transit œsophagien. - La technique utilisée initialement consiste à introduire dans les parois de l'œsophage, au niveau du cou, un microballonnet. Ce dernier obtenu par dilatation d'un cathéter est relié à un capteur de pression lui-même en liaison avec un enregistreur à papier. On enregistre ainsi les contractions œsophagiennes de déglutition au cours de la prise d'aliment ou d'eau. Nous obtenons de cette façon ef avec une très grande précision, à l'exception des quantités ingérées, la fréquence, la durée et le profil des repas.

B) Enregistrement par dispositif de pesée automatique de la mangeoire. - Ce dispositif permet de connaître, en plus des paramètres précédents, la dimension des repas unitaires. Dans ce but on utilise des balances à cadrans circulaires sur le plateau desquelles repose une mangeoire. Lors du déclenchement du repas, un ensemble hydraulique soulève la mangeoire et la présente rapidement au porc. Le repas terminé, le système hydraulique pose lentement la mangeoire sur le plateau de la balance. L'enregistrement des quantités ingérées s'effectue alors grâce à une caméra (caméras utilisant des films de $8 \mathrm{~m} / \mathrm{m}$ ou de $16 \mathrm{~m} / \mathrm{m}$ ) qui prend quelques images du cadran de la balance ainsi que d'une pendule placée en son centre. La commande de la caméra et de spots lumineux s'effectue grâce à des dispositifs électroniques reliés à des cellules photo-électriques à infra-rouge placées respectivement au-dessus ef légèrement en arrière de la mangeoire.

Le soulèvement de la mangeoire par les dispositifs hydrauliques protège la balance des chocs surtout lors de l'utilisation d'animaux d'un poids vif élevé. En fin de course, les bords de la mangeoire sont amenés au contact de systèmes de protection solidaires de la cage ce qui évite le gaspillage d'aliments.

Les cellules photo-électriques disposées dans le voisinage immédiat de la mangeoire sont reliées à un enregistreur papier. Ceci permet la mesure de la durée du repas. Nous évaluons également ce paramètre à l'aide de compteurs d'impulsions (une impulsion par seconde) placés au contact du cadran de la balance. Ces compteurs également reliés aux cellules photo-électriques possèdent la possibilité d'une remise à zéro automatique après chaque prise de vues.

Ces enregistrements par caméra, très précis, présentent quelques inconvénients tels que durée d'utilisation du film et le temps nécessaire à sa lecture. Aussi actuellement, nous utilisons également un autre dispositif permettant d'obtenir des résultats beaucoup plus rapidement.

C) Dispositif à disque. - Ce dispositif, constitué d'un disque à la périphérie duquel sont disposées des mangeoires, présente à l'animal et après chaque repas une nouvelle mangeoire. La rotation du disque s'effectue grâce à un ensemble électro-mécanique dont le fonctionnement est déclenché par des cellules photo-électriques placées respectivement au-dessus et immédiatement en arrière de la mangeoire. Cet appareillage, d'une grande fiabilité, ne prend en considération l'animal qu'après un temps 
de présence choisi au niveau de la mangeoire, évitant ainsi le vagabondage. De plus, la rotation du disque ne s'effectue qu'après un laps de temps affiché après la dernière prise d'aliments. On évite ainsi les mouvements intempestifs du disque lors de déplacement de va-et-vient que font certains animaux au cours du repas. De plus chez les porcelets après sevrage effectuant de très nombreux repas, cette commande retardée du disque fixe l'intervalle entre deux repas. Le critère d'un intervalle minimum de $10 \mathrm{~min}$ pour la séparation des repas a été choisi.

Un enregistreur papier relié aux cellules photo-électriques permet d'enregistrer l'heure, la durée du repas ef les intervalles entre repas ef leur fréquence.

L'observation des contractions œsophagiennes a montré que leur fréquence est sensiblement constante depuis le début à la fin du repas. Ceci indiquerait une vitesse d'ingestion constante pendant la prise d'aliment. Nous l'avons confirmé grâce à des observations de visu ef après des périodes de jeûne relativement courtes $(7-8 \mathrm{~h})$. Des appareillages nouveaux de commande des caméras et du disque permettent la prise en compte de ce paramètre supplémentaire pendant le déroulement normal de la séquence alimentaire.

D) Dispositif de mesure de la prise d'eau. - Deux électrodes de charbon placées au contact de l'eau d'un abreuvoir sont reliées à un dispositif électronique. Celui-ci est en connection d'une part avec un moteur électrique qui commande une pompe péristaltique, d'autre part, avec un deuxième dispositif électronique. Ce dernier effectue la mesure du numéro de passage de l'animal à l'abreuvoir, de la quantité d'eau bue au cours de celui-ci et de la quantité globale consommée au cours des différentes prises hydriques.

Le fonctionnement de cet ensemble qui alimente en eau l'abreuvoir au cours du repas se fait grâce à la rupture et au rétablissement du contact eau-électrodes de charbon. Le dispositif électronique de mesure esł relié à un enregistreur papier lequel prend en considération la durée et l'heure de la prise hydrique.

\section{Conditions expérimentales.}

Les animaux soumis au contrôle sont placés dans des cages individuelles, dont la taille varie avec le poids des animaux, elles-mêmes situées dans des locaux isolés. A l'intérieur de ces derniers, la température se trouve maintenue à $18^{\circ} \pm 1{ }^{\circ} \mathrm{C}$. Les porcs sont soumis à un cycle d'éclairement $12 / 12$ ( $7 \mathrm{~h}$ à $19 \mathrm{~h}$ ). L'aliment est distribué sous forme de granulés de composition suivante en p. 100 : orge 35 , blé 15 , son de blé 10 , tourteau de soja 18 , maïs 15 , sels minéraux et vitamines.

Trois races de porcs sont utilisées : des porcs Piétrain (4 animaux) souche maigre, des porcs Large White, d'obésité moyenne (13 animaux) et des porcs miniatures adultes qui présentent une nette tendance à l'obésité (4 animaux).

La séquence alimentaire des porcs Large-White (sevrés soit à 8 semaines, soit à 35 jours) et des porcs Piétrain (sevrés à 40 jours) a été enregistrée pendant des périodes de temps variables du sevrage à « l'état adulte ». En fin de croissance, seuls sont pris en considération les porcs mâles entiers.

Les porcs miniatures, âgés de 2 ans environ et dont le poids vif est compris entre 70 et $90 \mathrm{~kg}$ après adaptation à leurs nouvelles conditions d'ambiance pendant une quinzaine de jours, sont enregistrés pendant 3 semaines. 


\section{Résultats.}

Ils sont obtenus à partir des enregistrements du " profil alimentaire » de 21 animaux ef résumés dans les tableaux 1 et 2 .

a) Rythme journalier des prises. - Un animal soumis à un éclairement $12 / 12$ mange préférentiellement le jour. Ceci est observé chez tous les animaux des trois races. Ce caractère est d'autant plus net que l'on s'éloigne du sevrage : 60 p. 100 de l'ingéré quotidien sont situés en période diurne en début de croissance contre 65 p. 100 de l'ingéré quotidien en fin de croissance.

\section{TABLEAU 1}

Fréquence journalière par périodes de 15 jours à partir du sevrage des prises d'aliments et quantité consommée chez des porcs Piétrain ef Large White (L. W.)

\begin{tabular}{|c|c|c|c|c|c|c|c|}
\hline & & \multicolumn{2}{|c|}{ Ingéré global } & \multicolumn{2}{|c|}{ Ingéré par repas } & \multicolumn{2}{|c|}{ Nombre de repas } \\
\hline & & diurne & nocturne & diurne & nocturne & diurne & nocturne \\
\hline \multirow{3}{*}{$\begin{array}{l}\text { Période } \\
\text { No } 1\end{array}$} & Piétrain & $745^{3} \pm 132^{a}$ & $449^{3} \pm 21$ & - & - & $4,9^{3} \pm 1,1$ & $3,2^{3} \pm 0,1$ \\
\hline & $\begin{array}{l}\text { L. W. Sevrés } \\
60 \text { jours }\end{array}$ & $824^{5} \pm 92$ & $610^{5} \pm 78$ & $181^{2}$ & $158^{2}$ & $5,1^{5} \pm 0,3$ & $4,1^{5}+0,7$ \\
\hline & $\begin{array}{l}\text { L. W. Sevrés } \\
35 \text { jours }\end{array}$ & $542^{3} \pm 71$ & $345^{3} \pm 19$ & $86^{3} \pm 8$ & $78^{3} \pm 7$ & $6,8^{3} \pm 0,4$ & $4,3^{3} \pm 0,6$ \\
\hline \multirow{3}{*}{$\begin{array}{l}\text { Période } \\
N^{\circ} 2\end{array}$} & Piétrain & $870^{3} \pm 82$ & $497^{3} \pm 45$ & - & - & $4,8^{3} \pm 0,8$ & $2,7^{3} \pm 0,3$ \\
\hline & $\begin{array}{l}\text { L.W. Sevrés } \\
60 \text { jours }\end{array}$ & $1212^{5} \pm 79$ & $813^{5} \pm 96$ & $276^{2}$ & $258^{2}$ & $4,9^{5} \pm 0,3$ & $3,4^{5} \pm 0,3$ \\
\hline & $\begin{array}{l}\text { L. W. Sevrés } \\
35 \text { jours }\end{array}$ & $924^{3} \pm 53$ & $437^{3} \pm 104$ & $147^{3} \pm 11$ & $143^{3} \pm 4$ & $5,9^{3} \pm 0,5$ & $3,0^{3} \pm 0,6$ \\
\hline \multirow{3}{*}{$\begin{array}{l}\text { Période } \\
\text { No } 3\end{array}$} & Piétrain & $968^{3} \pm 90$ & $621^{3} \pm 8$ & - & - & $4,2^{3} \pm 0,7$ & $2,8^{3} \pm 0,2$ \\
\hline & $\begin{array}{l}\text { L. W. Sevrés } \\
60 \text { jours }\end{array}$ & $1367^{4} \pm 85$ & $947^{4} \pm 162$ & $341^{1}$ & $376^{1}$ & $4,6^{4} \pm 0,4$ & $3,0^{4} \pm 0,2$ \\
\hline & $\begin{array}{l}\text { L. W. Sevrés } \\
35 \text { jours }\end{array}$ & $1360^{4} \pm 106$ & $469^{4} \pm 48$ & $217^{4} \pm 24$ & $191^{4} \pm 32$ & $6,1^{4} \pm 0,3$ & $2,2^{4} \pm 0,4$ \\
\hline \multirow{3}{*}{$\begin{array}{l}\text { Période } \\
\text { No } 4\end{array}$} & Piétrain & $1049^{3} \pm 132$ & $566^{3} \pm 15$ & - & - & $3,8^{3} \pm 0,5$ & $2,4^{3} \pm 0,1$ \\
\hline & $\begin{array}{l}\text { L. W. Sevrés } \\
60 \text { jours }\end{array}$ & $1641^{4} \pm 260$ & $829^{4} \pm 120$ & $496^{1}$ & $340^{1}$ & $4,0^{4} \pm 0,7$ & $2,4^{4} \pm 0,3$ \\
\hline & $\begin{array}{l}\text { L. W. Sevrés } \\
35 \text { jours }\end{array}$ & $1561^{1}$ & $366^{1}$ & $303^{1}$ & $162^{1}$ & $5,4^{1}$ & $2,2^{1}$ \\
\hline \multirow{3}{*}{$\begin{array}{l}\text { Période } \\
\text { No } 5\end{array}$} & Piétrain & $1144^{3} \pm 49$ & $947^{3} \pm 126$ & - & - & $3,4^{3} \pm 0,3$ & $2,9^{3} \pm 0,4$ \\
\hline & $\begin{array}{l}\text { L. W. Sevrés } \\
60 \text { jours }\end{array}$ & $1884^{3} \pm 229$ & $903^{3} \pm 158$ & $502^{1}$ & $437^{1}$ & $4,4^{3} \pm 0,7$ & $2,1^{3} \pm 0,2$ \\
\hline & $\begin{array}{l}\text { L. W. Sevrés } \\
35 \text { jours }\end{array}$ & $1586^{3} \pm 228$ & $331^{3} \pm 67$ & $337^{3} \pm 51$ & $210^{3} \pm 7$ & $5,0^{3} \pm 0$ & $1,7^{3} \pm 0,3$ \\
\hline
\end{tabular}


TABLEAU 1 (suite)

\begin{tabular}{|c|c|c|c|c|c|c|c|}
\hline & & \multicolumn{2}{|c|}{ Ingéré global } & \multicolumn{2}{|c|}{ Ingéré par repas } & \multicolumn{2}{|c|}{ Nombre de repas } \\
\hline & & diurne & nocturne & diurne & nocturne & diurne & nocturne \\
\hline & Piétrain & $1659^{2}$ & $1075^{2}$ & - & - & $8,5^{2}$ & $5,0^{2}$ \\
\hline \multirow[t]{3}{*}{$\begin{array}{l}\text { Période } \\
\text { No } 6\end{array}$} & $\begin{array}{l}\text { L. W. Sevrés } \\
60 \text { jours }\end{array}$ & $1750^{2}$ & $1050^{2}$ & - & - & $2,9^{2}$ & $1,7^{2}$ \\
\hline & L. W. Sevrés & 一 & - & - & - & - & - \\
\hline & Piétrain & - & - & - & - & - & - \\
\hline \multirow[t]{2}{*}{$\begin{array}{l}\text { Période } \\
\text { No } 7\end{array}$} & $\begin{array}{l}\text { L. W. Sevrés } \\
60 \text { jours }\end{array}$ & - & - & - & - & - & - \\
\hline & $\begin{array}{l}\text { L. W. Sevrés } \\
35 \text { jours }\end{array}$ & $1686^{1}$ & $1332^{1}$ & - & - & $2,0^{1}$ & $1,4^{1}$ \\
\hline \multirow{3}{*}{$\begin{array}{l}\text { Période } \\
\text { No } 8\end{array}$} & Piétrain & - & - & - & - & - & - \\
\hline & $\begin{array}{l}\text { L. W. Sevrés } \\
60 \text { jours }\end{array}$ & - & - & - & - & - & - \\
\hline & $\begin{array}{l}\text { L. W. Sevrés } \\
35 \text { jours }\end{array}$ & $1683^{1}$ & 11211 & - & - & $2,2^{1}$ & $1,0^{1}$ \\
\hline \multirow{3}{*}{$\begin{array}{l}\text { Période } \\
\text { No9 }\end{array}$} & Piétrain & - & - & 一 & - & - & - \\
\hline & $\begin{array}{l}\text { L. W. Sevrés } \\
60 \text { jours }\end{array}$ & - & - & - & - & - & - \\
\hline & $\begin{array}{l}\text { L. W. Sevrés } \\
35 \text { jours }\end{array}$ & $2244^{1}$ & $1153^{1}$ & - & - & $2,0^{1}$ & $1,0^{1}$ \\
\hline \multirow{3}{*}{$\begin{array}{l}\text { Période } \\
\text { No } 10\end{array}$} & Piétrain & - & - & - & - & - & - \\
\hline & $\begin{array}{l}\text { L. W. Sevrés } \\
60 \text { jours }\end{array}$ & - & - & - & - & - & - \\
\hline & $\begin{array}{l}\text { L. W. Sevrés } \\
35 \text { jours }\end{array}$ & $2201^{1}$ & $1191^{1}$ & - & - & $2,0^{1}$ & $1,0^{1}$ \\
\hline
\end{tabular}

L'exposant affecté à la moyenne représente le nombre d'animaux pris en considération.

$a$ : Ecart-type de la moyenne.

Chez les porcs Piétrain et Large White, la fréquence des prises est différente le jour et la nuit. Les animaux font davantage de repas le jour : 59 p. 100 du nombre total quotidien de repas en début de croissance contre 67 p. 100 en fin de croissance. La fréquence quotidienne des prises diminue au fur et à mesure de la croissance. Elle est, en moyenne, de 9,5 repas au cours des premières semaines après le sevrage ef seulement de 3 repas en fin de croissance. Ce « pattern adulte » observé pour 6 porcs l'est actuellement chez 2 autres porcs Large-White et 1 porc Piétrain en cours d'expérience. Il semble d'après ce petit nombre d'animaux qu'il apparaisse vers les 6-7e mois d'âge. 
TABLEAU 2

Fréquence journalière, par périodes de 8 jours des prises d'aliments ef quantité consommée chez des porcs miniatures ef un porc Lorge White (L. W.)

\begin{tabular}{|c|c|c|c|c|c|c|c|}
\hline & & \multicolumn{2}{|c|}{ Ingéré global } & \multicolumn{2}{|c|}{ Ingéré par repas } & \multicolumn{2}{|c|}{ Nombre de repas } \\
\hline & & diurne & nocturne & diurne & nocturne & diurne & nocłurne \\
\hline \multirow{2}{*}{$\begin{array}{l}\text { Période } \\
\text { No } 1\end{array}$} & $\begin{array}{l}\text { Miniafure } \\
\text { adulte }\end{array}$ & $1303^{4} \pm 177^{a}$ & $843^{4} \pm 96$ & - & - & $4,1^{4} \pm 0,7$ & $2,8^{4} \pm 0,3$ \\
\hline & L. W. adulte & $2872^{1}$ & $1196^{1}$ & $1504^{1}$ & 12321 & $1,9^{1}$ & $0,8^{1}$ \\
\hline \multirow{2}{*}{$\begin{array}{l}\text { Période } \\
\text { No } 2\end{array}$} & $\begin{array}{l}\text { Miniature } \\
\text { adulte }\end{array}$ & $1414^{4} \pm 117$ & $780^{4} \pm 75$ & - & - & $4,1^{3} \pm 0,5$ & $2,7^{3} \pm 0,3$ \\
\hline & L. W. adulte & $2644^{1}$ & $1420^{1}$ & $1617^{1}$ & 16891 & $1,7^{1}$ & $0,8^{1}$ \\
\hline \multirow{2}{*}{$\begin{array}{l}\text { Période } \\
\text { No } 3\end{array}$} & $\begin{array}{l}\text { Miniature } \\
\text { adulte }\end{array}$ & $1433^{3} \pm 144$ & $719^{3} \pm 109$ & - & - & $4,2^{3} \pm 0,1$ & $2,9^{3} \pm 0,7$ \\
\hline & L. W. adulte & - & - & - & - & - & - \\
\hline
\end{tabular}

L'exposant affecté à la moyenne représente le nombre d'animaux pris en considération.

a : Ecart-type de la moyenne.

b) Importance des prises. - La valeur des prises est en moyenne 23 p. 100 supérieure lorsque l'animal est éclairé.

L'amplitude des repas augmente au fur et à mesure de la croissance : en moyenne $127 \mathrm{~g}$ en début de croissance contre $394 \mathrm{~g} 2,5$ mois plus tard.

Chez les porcs miniatures adultes la fréquence des prises est nettement plus élevée que celle des porcs Large White en fin de croissance $(6,8$ repas quotidiens contre $3,0)$. Cette prise d'aliments est toujours plus élevée le jour $=64$ p. 100 de l'ingéré quotidien.

La relation entre amplitude du repas et intervalle qui suit (corrélation postprandiale) a été examinée, au cours de la période d'éclairement, chez 6 porcs présentant le « pattern adulte » ( 2 repas le jour, 1 repas la nuit) el chez lesquels a été prise en compte la durée des repas. Les coefficients de corrélation obtenus à partir des données brutes considérées isolément sonf les suivants :

$\begin{array}{llll}\text { Porc } n^{\circ} 81189 & r=0,77 & \mathrm{DL}=17 * * & \mathrm{P}<0,01 \\ \text { Porc } n^{\circ} 81268 & r=0,53 & \mathrm{DL}=18 * & \mathrm{P}<0,05 \\ \text { Porc } n^{\circ} 75602 & r=0,49 & \mathrm{DL}=31 * * & \\ \text { Porc } n^{\circ} 93050 & \mathrm{r}=0,28 & \mathrm{DL}=19 \mathrm{NS} & \\ \text { Porc } n^{\circ} 77917 & \mathrm{r}=0,17 & \mathrm{DL}=20 \mathrm{NS} & \\ \text { Porc } n^{\circ} 75588 & \mathrm{r}=0,07 & \mathrm{DL}=19 \mathrm{NS} & \end{array}$

Les données sur la prise hydrique ne sont pas disponibles, les appareillages de mesure étant de conception récente. 


\section{Discussion et conclusions.}

Nous venons de décrire des dispositifs de mesure précis de la prise alimentaire de porcs de trois souches différentes et de poids très variable. L'utilisation d'appareillages tels que les systèmes hydrauliques, les dispositifs de commande des caméras, des spots lumineux, ne perturbent pas le déroulement normal de la séquence alimentaire du porc alimenté à volonté. Ceci a été vérifié en utilisant la technique d'enregistrement de la motricité œsophagienne, totalement silencieuse, seule ou couplée à ces dispositifs.

Comme chez le rat, on observe que la consommation se répartit inégalement entre les périodes diurnes et nocturnes. Mais contrairement à ce dernier, le porc consomme davantage le jour que la nuit.

Chez le porc en fin de croissance et à l'état adulte, les prises d'aliments sont très bien individualisées et importantes. On peut ainsi déterminer sans équivoque la durée de l'intervalle précédant ou suivant chaque repas ainsi que leur amplitude.

Pour le même animal et, sur de courtes périodes, la distribution des repas est assez reproductible d'un jour à l'autre. Toutefois, ce « pattern » diffère selon les souches et d'un animal à un autre. On observe donc, comme l'ont montré par ailleurs Le Magnen et Tallon (1966) chez le rat, des « patterns » individuels et non un « pattern » stéréotypé. Ceci montre également que les différents appareillages utilisés ne perturbent pas le comportement alimentaire du porc.

Les vitesses d'ingestion, généralement constantes, enregistrées au cours de repas effectués après une période de jeûne relativement courte (8-10 h) devront être mesurées à l'aide d'appareillages précités chez l'animal nourri à volonté.

Un désaccord ressort des données de la bibliographie sur l'existence d'une relafion entre la dimension des repas et l'intervalle entre repas. Le Magnen et Tallon (1966) signalent une haute corrélation entre l'amplitude d'un repas et l'intervalle qui le sépare du suivant (corrélation post-prandiale) chez le rat alimenté ad libitum. Pour ces auteurs, l'intervalle entre deux repas serait fonction de la quantité de métabolites ingérés lors du repas qui précède. Cette observation confirmée par certains auteurs est rejetée par d'autres chez le rat ou d'autres espèces. Une corrélation préprandiale est rapportée par De Castro et Balagura (1975) chez le rat en croissance.

Chez la moitié des porcs étudiés en fin de croissance, on note une corrélation post-prandiale positive.

Chez le porcelet, Montgomery, Flux et Carr (1978) signalent des corrélations post-prandiale et pré-prandiale faibles. Notons que ces auteurs utilisent des animaux très jeunes dont les prises d'aliments sont très nombreuses et nécessitent le choix d'un critère pour distinguer deux repas (ici intervalles d'au moins 10 min sans consommation).

Les résultats préliminaires acquis au cours de ce travail d'exploration ne permettent pas de tirer des conclusions définitives. Nous devrons examiner si une corrélation post-prandiale existe chez les porcs miniatures dont la durée d'ingestion aura été prise en compte et, chez les animaux des autres souches, plus nombreux et plus âgés. L'existence possible d'une corrélation pré-prandiale sera recherchée chez les ani- 
maux en croissance dont la séquence alimentaire aura été enregistrée pendant des périodes de temps suffisantes.

Les porcs de race Large White ou Piétrain, dont la séquence alimentaire en fin de croissance est très bien définie, avec 2 repas le jour et 1 seul repas la nuit séparés par des intervalles importants, nous semblent représenter un animal de choix dans l'étude des mécanismes intervenant dans le déclenchement et l'arrêł d'un repas. Ce modèle expérimental est actuellement en cours d'exploitation.

Journées Ingestion-Digestion-Absorption de I'Association française de Nutrition, Paris, 15-16 novembre 1979.

Remerciements. - Nous remercions MM. Barbrel, Martin et Linot, de la Station de Physiologie animale (INRA), ainsi que MM. Geandier ef Mange, Professeurs d'électronique au Lycée Technique de Versailles et leurs élèves pour la mise au point ef la réalisation des différents appareillages.

\section{Références}

AUFFRAY P., 1969. Effets des lésions des noyaux ventro-médians hypothalamiques sur la prise d'aliments chez le porc. Ann. Biol. anim. Bioch. Biophys., 9, 513-526.

AUFFRAY P., BAHY C., MARCILLOUX J. C., 1974. Analyse des séquences alimentaires du porc nourri ad libilum. Journées Rech. Porcine en France, I.N.R.A.-I.T.P. ed., Paris, 277-281.

DE CASTRO J. M., BALAGURA S., 1975. Ontogeny of meal patterning in rats and its recapitulation during recovery from lateral hypothalamic lesions. J. compar. physiol. Psychol., 89N, 791-802.

FOWLER V. R., Mc DONALD A., ROBB I., 1971. The measurement of diurnal patterns of feed intake in the pig. Anim. Prod., 13, 378.

KHALAF F., 1969. Hyperphagia and aphagia in swine with induced hypothalamic lesions. Res. vet. Sci., 10, 514-517.

LE MAGNEN J., TALLON S., 1966. La périodicité spontanée de la prise d'aliments ad libitum du rat blanc. J. Physiol., 58, 323-349.

MONTGOMERY G. W., FLUX D. S., CARR J. R., 1978. Feedings patterns in pigs : the effects of amino acid deficiency. Physiol. Behov., 20, 693-698. 